\section{Primate studies: hear the public's views}

A painful irony in the disrupted flow of primates to US research labs (Nature 483, 381-382; 2012) is that the number being used in laboratory experiments is at an all-time high.

If the scientific community is to maintain the support and trust of the public, which funds much of its work, then research practices and policies should change to reflect society's views on what constitutes the ethical treatment of animals. These changes need to be speeded up.

Committees that review and approve animal experiments at US facilities should not be dominated by those who work in animal labs and have vested interests in continuing animal research (L. A. Hansen et al. Animals 2, 68-75; 2012). There are too few members of the public on these US committees, and those who are involved say that they are often marginalized. In other countries, such as Sweden and Australia, half or one-third of committees must comprise non-scientists and animal-welfare representatives.

If scientists continue to disregard the substantial and growing public opposition to harmful research on primates and other animals, more protest campaigns are inevitable.

Lawrence A. Hansen University of California, San Diego, La Jolla, California, USA.

lahansen@ucsd.edu

\section{Primate studies: fix welfare issues first}

Record numbers of non-human primates are being used in US labs, so it is unlikely that limiting imports will hold back vital areas of research as you imply (Nature 483, 381-382; 2012).

A report from the American Anti-Vivisection Society (AAVS; see go.nature.com/ gbqlel) indicates that imports of monkeys born to wild-caught parents quadrupled during 1998-2008. Conservationists are concerned about global trade in crab-eating macaques (Macaca fascicularis), the import of which has doubled in recent years.

Scientists must urgently address the extreme animalwelfare issues surrounding these imports. The AAVS report, which is based on information from US federal agencies and scientific studies, has revealed that monkeys destined for US labs typically endure long, gruelling air and land transportation; entire groups have been killed after quarantine on testing positive for tuberculosis; many die from transport injuries or stress in quarantine; and survivors show negative physiological and behavioural effects for several months after the journey.

More airlines are likely to back away from a dirty job that they are ill-equipped to do properly. Crystal Miller-Spiegel American Anti-Vivisection Society, Jenkintown, Pennsylvania, USA. cmillerspiegel@aavs.org

\section{Primate studies: trials don't always translate}

In your discussion on the campaign against animal research (Nature 483, 373-374; 2012), you mention a study in macaques that has moved into early clinical trials in humans, with promising results. Sadly, there is a yawning chasm between early promise in trials and efficacy.

The US Food and Drug Administration reports that more than $90 \%$ of trials fail (see go.nature.com/h2365q), even though the treatments tested, by definition, were considered sufficiently efficacious and safe in animals to merit a clinicaltrial licence.

Many other treatments to protect the brain after stroke have failed in humans, despite success in rodent and primate trials (V. E. Collins et al. Ann. Neurol. $59,467-477 ; 2006)$. None of the 85 or so candidate HIV vaccines that were effective in primates has so far worked in humans (J. Bailey Altern. Lab. Anim. 36, 381-428; 2008). The monoclonal antibody that caused severe inflammatory reactions in a 2006 clinical trial at Northwick Park Hospital, London, caused no problems in primates at 500 times the dose given to the human volunteers.

The public is rightly concerned about the transportation of primates for questionable experimental purposes. These cannot justify the degree of suffering involved during capture, in breeding and holding facilities and during lengthy transportation (see go.nature. com/svbuvj).

Michelle Thew British Union for the Abolition of Vivisection, London, UK.

michelle.thew@buav.org

\section{Higgs can claim name of massive boson}

Attempts to rule against naming the Higgs boson after physicist Peter Higgs suggest that political correctness is taking over from scholarship (Nature 483, 374; 2012). Your suggestion that the name Higgs should be retained for reasons akin to business branding is hardly better. Higgs has a unique claim to the massive boson in question.

My book The Infinity Puzzle (Oxford Univ. Press, 2011) covers the history of the Higgs hypothesis in detail. It is true that Higgs is one of several theorists who, in 1964, independently discovered how to give mass to fundamental particles, and that it would be inappropriate to refer to the hypothesis of mass generation as the 'Higgs mechanism'. However, it was Higgs alone who drew attention to the massive boson whose detection can prove the hypothesis. So naming the boson after him, as Ben Lee did in 1972, is justifiable.

Frank Close University of

Oxford, Oxford, UK.

f.close1@physics.ox.ac.uk

\section{More credit due to India's scientists}

Any increase in India's science budget for 2012-13 is likely to be wiped out by a $5-10 \%$ rise in the cost of research commodities, owing to the country's high rate of inflation (Nature 483, 384; 2012). Neither will the modest extra funding tackle the glut of unemployed science $\mathrm{PhD}$ graduates (for example, around $60 \%$ of female science PhDs do not have a research position).

The reasons for this situation are not just economic. In my opinion, India's policymakers are failing to recognize scientists' achievements. In May last year, for example, environment minister Jairam Ramesh intimated that India's elite institutions, which include the Indian Institutes of Technology and of Management, fall short of world-class standards; the head of the prime minister's scientific advisory council, C. N. R. Rao, seems to agree with this view (see go.nature.com/snnbbt).

Yet India is ranked 11th in the world by number of publications and 16th on the basis of total citations during the past 10 years. Many of these publications were in leading international journals. Instead of squandering this talent, the government should provide the incentive and the means for the nation to fulfil its potential. Jagadeesh Bayry Institut National de la Santé et de la Recherche Médicale, Paris, France. jagadeesh.bayry@crc.jussieu.fr

CONTRIBUTIONS

Correspondence may be sent to correspondence@ nature.com after consulting the author guidelines at go.nature.com/cmchno. Alternatively, readers may comment online: www. nature.com/nature. 